\title{
MINIMAL AND MAXIMAL SOLUTIONS TO SYSTEMS OF DIFFERENTIAL EQUATIONS WITH A SINGULAR MATRIX
}

\author{
TADEUSZ JANKOWSKI ${ }^{1}$
}

(Received 2 July, 2001; revised 15 February, 2002)

\begin{abstract}
The monotone iterative technique is applied to a system of ordinary differential equations with a singular matrix. The existence of extremal solutions is proved.
\end{abstract}

\section{Introduction}

Many problems arising in the physical sciences, engineering, biology and applied mathematics lead to mathematical models described by systems of differential equations with initial conditions of the form

$$
x^{\prime}(t)=f_{1}(t, x(t)), \quad t \in J=[0, T], x(0)=x_{0} \in \mathbb{R}^{p},
$$

where $f_{1} \in C\left(J \times \mathbb{R}^{p}, \mathbb{R}^{p}\right)$. Conditions on $f_{1}$ which guarantee the existence of solutions of problem (1.1) are important analysis theorems. To show that problem (1.1) has a solution, one can employ fixed point theorems (Banach, Schauder), the Leray-Schauder theory of topological degree or the method of successive iterations. Assuming that $f_{1}$ satisfies the Lipschitz condition with respect to the last variable one can show that problem (1.1) has a unique solution. If we assume that $f_{1}$ satisfies only a one-sided Lipschitz condition, then we can show that problem (1.1) has extremal solutions. Such a result can be obtained when the method of upper and lower solutions is used. This interesting and fruitful technique for proving existence results shows that corresponding monotone sequences converge to the minimal and maximal solutions of our problem (there are some applications of this technique, for example, in [3]). The constructive proofs of existence also provide numerical procedures for the computation

\footnotetext{
${ }^{1}$ Technical University of Gdańsk, Department of Differential Equations, $11 / 12$ G. Narutowicz Str., 80-952 Gdańsk, Poland; e-mail: tjank@mifgate.mif.pg.gda.pl.

(C) Australian Mathematical Society 2003, Serial-fee code 1446-1811/03
} 
of solutions. Problem (1.1) may be generalised by adding an algebraic system to obtain the differential-algebraic system

$$
\begin{cases}x^{\prime}(t)=f_{1}(t, x(t), y(t)), & t \in J, x(0)=x_{0} \\ y(t)=f_{2}(t, x(t), y(t)), & t \in J .\end{cases}
$$

Note that the last system is a special case of a problem discussed in this paper, namely

$$
\left\{\begin{array}{l}
A x^{\prime}(t)=f\left(t, x(t), x^{\prime}(t)\right), \quad t \in J=[0, T], \\
x(0)=k_{0} \in \mathbb{R}^{m},
\end{array}\right.
$$

where $f \in C\left(J \times \mathbb{R}^{m} \times \mathbb{R}^{m}, \mathbb{R}^{m}\right)$ and $A$ is a singular square matrix of order $m$. Note that problem (1.2) is identical to

$$
x^{\prime}(t)=(A+B)^{-1}\left[f\left(t, x(t), x^{\prime}(t)\right)+B x^{\prime}(t)\right], \quad t \in J, x(0)=k_{0}
$$

provided that the matrix $B$ is a square matrix of order $m$ such that $A+B$ is nonsingular.

It is well-known that the method of lower and upper solutions coupled with the monotone iterative technique provides a practical tool to generate monotone sequences that converge to extremal solutions (see [1], see also [2-8]). The purpose of this paper is to extend this technique to problems of type (1.2). This method is useful since any member of the corresponding linear monotone iterations is an approximate solution of (1.2). In our discussion, we assume that $f$ satisfies a one-sided Lipschitz condition showing that problem (1.2) has extremal solutions. Note that the system of differential-algebraic equations is a special case of (1.2). Some examples are also given.

\section{Main results}

A function $v \in C^{1}\left(J, \mathbb{R}^{m}\right)$ is said to be a lower solution of problem (1.2) if

$$
\left\{\begin{array}{l}
A v^{\prime}(t) \leq f\left(t, v(t), v^{\prime}(t)\right), \quad t \in J, \\
v(0) \leq k_{0}
\end{array}\right.
$$

and an upper solution of (1.2) if the above inequalities are reversed. In this paper, the vectorial inequalities mean that the same inequalities hold between their corresponding components. Note that if the matrix $(A+B)^{-1}$ exists, $(A+B)^{-1} \geq 0$ and $v$ is a lower solution of problem (1.2), then $v$ satisfies the relations

$$
\left\{\begin{array}{l}
v^{\prime}(t) \leq(A+B)^{-1}\left[f\left(t, v(t), v^{\prime}(t)\right)+B v^{\prime}(t)\right], \quad t \in J \\
v(0) \leq k_{0}
\end{array}\right.
$$

Here $(A+B)^{-1} \geq 0$ means that some entries of $(A+B)^{-1}$ may be equal to zero.

The next lemma is a special case of $[4$, Theorem 1.1.4]. 
LEMMA 2.1. Assume that $d_{i j}(t) \geq 0, t \in J$ for $i \neq j$, where $D=\left[d_{i j}\right]$ is a continuous square matrix of order $m$. Let

Then $p(t) \leq 0$ on $J$.

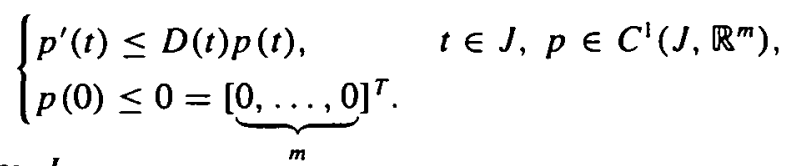

Let us define the following set:

$$
\Omega=\left\{(t, u, v): t \in J, y_{0}(t) \leq u \leq z_{0}(t), y_{0}^{\prime}(t) \leq v \leq z_{0}^{\prime}(t), u, v \in \mathbb{R}^{m}\right\},
$$

where $y_{0}, z_{0} \in C^{1}\left(J, \mathbb{R}^{m}\right)$.

Now we are in a position to show the following existence result.

THEOREM 2.2. Assume that $f \in C\left(\Omega, \mathbb{R}^{m}\right)$ and

(i) $y_{0}, z_{0} \in C^{l}\left(J, \mathbb{R}^{m}\right)$ are lower and upper solutions of (1.2), respectively, and such that $y_{0}(t) \leq z_{0}(t)$ and $y_{0}^{\prime}(t) \leq z_{0}^{\prime}(t)$ on $J$;

(ii) there exists a square matrix $B$ of order $m$ such that $(A+B)^{-1}$ exists, $(A+$ $B)^{-1} \geq 0$, and the condition $f(t, u, \alpha)-f(t, u, \bar{\alpha}) \leq B[\bar{\alpha}-\alpha]$ holds for $y_{0}(t) \leq$ $u \leq z_{0}(t)$ and $y_{0}^{\prime}(t) \leq \alpha \leq \bar{\alpha} \leq z_{0}^{\prime}(t), t \in J$;

(iii) there exists a square matrix $N$ of order $m$ such that $N \geq 0$, and for $y_{0}(t) \leq$ $u \leq \bar{u} \leq z_{0}(t), t \in J$, it holds that $f(t, u, \alpha)-f(t, \bar{u}, \alpha) \leq-N[\bar{u}-u]$.

Then there exist monotone sequences $\left\{y_{n}\right\}$ and $\left\{z_{n}\right\}$ such that $y_{n}(t) \rightarrow y(t)$ and $z_{n}(t) \rightarrow z(t)$ on $J$ as $n \rightarrow \infty$ and this convergence is uniform and monotonic on $J$. Moreover the functions $y$ and $z$ are minimal and maximal solutions of problem (1.2), respectively.

ProOF. We construct the sequences $\left\{y_{n}\right\}$ and $\left\{z_{n}\right\}$ using the formulas

$$
\begin{cases}y_{n+1}^{\prime}(t)=(A+B)^{-1}\left\{f\left(t, y_{n}, y_{n}^{\prime}\right)+B y_{n}^{\prime}(t)+N\left[y_{n+1}(t)-y_{n}(t)\right]\right\}, & y_{n+1}(0)=k_{0}, \\ z_{n+1}^{\prime}(t)=(A+B)^{-1}\left\{f\left(t, z_{n}, z_{n}^{\prime}\right)+B z_{n}^{\prime}(t)+N\left[z_{n+1}(t)-z_{n}(t)\right]\right\}, & z_{n+1}(0)=k_{0} .\end{cases}
$$

First of all, we are going to show the following relation:

$$
\left\{\begin{array}{l}
y_{0}(t) \leq y_{1}(t) \leq z_{1}(t) \leq z_{0}(t) \\
y_{0}^{\prime}(t) \leq y_{1}^{\prime}(t) \leq z_{1}^{\prime}(t) \leq z_{0}^{\prime}(t), \quad t \in J
\end{array}\right.
$$

Put $p=y_{0}-y_{1}$ on $J$. Then $p(0) \leq 0$. Since $(A+B)^{-1} \geq 0$, by assumption (i) we have

$$
\begin{aligned}
p^{\prime}(t) & \leq(A+B)^{-1}\left\{f\left(t, y_{0}, y_{0}^{\prime}\right)+B y_{0}^{\prime}(t)-f\left(t, y_{0}, y_{0}^{\prime}\right)-B y_{0}^{\prime}(t)-N\left[y_{1}(t)-y_{0}(t)\right]\right\} \\
& =(A+B)^{-1} N p(t)
\end{aligned}
$$


By Lemma 2.1, we have $p(t) \leq 0$ and then $p^{\prime}(t) \leq 0$ on $J$ showing that $y_{0}(t) \leq y_{1}(t)$, $y_{0}^{\prime}(t) \leq y_{1}^{\prime}(t), t \in J$. Similarly, we can show that $z_{1}(t) \leq z_{0}(t), z_{1}^{\prime}(t) \leq z_{0}^{\prime}(t), t \in J$.

Put $p=y_{1}-z_{1}$, so $p(0)=0$. Then, by (ii) and (iii), we have

$$
\begin{aligned}
p^{\prime}(t)= & (A+B)^{-1}\left\{f\left(t, y_{0}, y_{0}^{\prime}\right)-f\left(t, z_{0}, y_{0}^{\prime}\right)+f\left(t, z_{0}, y_{0}^{\prime}\right)-f\left(t, z_{0}, z_{0}^{\prime}\right)\right. \\
& \left.-B\left[z_{0}^{\prime}(t)-y_{0}^{\prime}(t)\right]+N\left[y_{1}(t)-y_{0}(t)-z_{1}(t)+z_{0}(t)\right]\right\} \\
\leq & (A+B)^{-1}\left\{-N\left[z_{0}(t)-y_{0}(t)\right]+B\left[z_{0}^{\prime}(t)-y_{0}^{\prime}(t)\right]\right. \\
& \left.+B\left[y_{0}^{\prime}(t)-z_{0}^{\prime}(t)\right]+N\left[y_{1}(t)-y_{0}(t)-z_{1}(t)+z_{0}(t)\right]\right\} \\
= & (A+B)^{-1} N p(t), \quad t \in J .
\end{aligned}
$$

Hence we have $p(t) \leq 0$ and then $p^{\prime}(t) \leq 0$ on $J$ showing that $y_{1}(t) \leq z_{1}(t)$ and $y_{1}^{\prime}(t) \leq z_{1}^{\prime}(t), t \in J$. Thus (2.1) holds.

In the next step we need to show that $y_{1}$ and $z_{1}$ are lower and upper solutions of problem (1.2), respectively. Then, by assumptions (ii) and (iii), we obtain

$$
\begin{aligned}
A y_{1}^{\prime}(t)= & f\left(t, y_{0}, y_{0}^{\prime}\right)+B\left[y_{0}^{\prime}(t)-y_{1}^{\prime}(t)\right]+N\left[y_{1}(t)-y_{0}(t)\right] \\
& -f\left(t, y_{1}, y_{0}^{\prime}\right)+f\left(t, y_{1}, y_{0}^{\prime}\right)-f\left(t, y_{1}, y_{1}^{\prime}\right)+f\left(t, y_{1}, y_{1}^{\prime}\right) \\
\leq & f\left(t, y_{1}, y_{1}^{\prime}\right)-N\left[y_{1}(t)-y_{0}(t)\right] \\
& +B\left[y_{1}^{\prime}(t)-y_{0}^{\prime}(t)\right]+B\left[y_{0}^{\prime}(t)-y_{1}^{\prime}(t)\right]+N\left[y_{1}(t)-y_{0}(t)\right] \\
= & f\left(t, y_{1}, y_{1}^{\prime}\right)
\end{aligned}
$$

and

$$
\begin{aligned}
A z_{1}^{\prime}(t)= & f\left(t, z_{0}, z_{0}^{\prime}\right)+B\left[z_{0}^{\prime}(t)-z_{1}^{\prime}(t)\right]+N\left[z_{1}(t)-z_{0}(t)\right]-f\left(t, z_{1}, z_{0}^{\prime}\right) \\
& +f\left(t, z_{1}, z_{0}^{\prime}\right)-f\left(t, z_{1}, z_{1}^{\prime}\right)+f\left(t, z_{1}, z_{1}^{\prime}\right) \\
\geq & f\left(t, z_{1}, z_{1}^{\prime}\right)+N\left[z_{0}(t)-z_{1}(t)\right]-B\left[z_{0}^{\prime}(t)-z_{1}^{\prime}(t)\right] \\
& +B\left[z_{0}^{\prime}(t)-z_{1}^{\prime}(t)\right]+N\left[z_{1}(t)-z_{0}(t)\right] \\
= & f\left(t, z_{1}, z_{1}^{\prime}\right)
\end{aligned}
$$

showing that $y_{1}$ and $z_{1}$ are lower and upper solutions of problem (1.2), respectively.

For some $k \geq 1$, let us assume that

$$
\begin{cases}y_{k-1}(t) \leq y_{k}(t) \leq z_{k}(t) \leq z_{k-1}(t), & t \in J, \\ y_{k-1}^{\prime}(t) \leq y_{k}^{\prime}(t) \leq z_{k}^{\prime}(t) \leq z_{k-1}^{\prime}(t), & t \in J,\end{cases}
$$

and let $y_{k}$ and $z_{k}$ be lower and upper solutions of problem (1.2), respectively. We shall prove that

$$
\begin{cases}y_{k}(t) \leq y_{k+1}(t) \leq z_{k+1}(t) \leq z_{k}(t), & t \in J \\ y_{k}^{\prime}(t) \leq y_{k+1}^{\prime}(t) \leq z_{k+1}^{\prime}(t) \leq z_{k}^{\prime}(t), & t \in J\end{cases}
$$


Put $p=y_{k}-y_{k+1}$. Then

$$
\begin{aligned}
p^{\prime}(t) & \leq(A+B)^{-1}\left(f\left(t, y_{k}, y_{k}^{\prime}\right)+B y_{k}^{\prime}(t)-f\left(t, y_{k}, y_{k}^{\prime}\right)-B y_{k}^{\prime}(t)-N\left[y_{k+1}(t)-y_{k}(t)\right]\right\} \\
& =(A+B)^{-1} N p(t)
\end{aligned}
$$

with $p(0)=0$. Hence, by Lemma 2.1,p(t) $\leq 0$ and $p^{\prime}(t) \leq 0, t \in J$, showing that $y_{k}(t) \leq y_{k+1}(t)$ and $y_{k}^{\prime}(t) \leq y_{k+1}^{\prime}(t), t \in J$. Using the same argument we can prove that $z_{k+1}(t) \leq z_{k}(t)$ and $z_{k+1}^{\prime}(t) \leq z_{k}^{\prime}(t), t \in J$.

Let $p=y_{k+1}-z_{k+1}$, so $p(0)=0$. Then we get

$$
\begin{aligned}
p^{\prime}(t)= & (A+B)^{-1}\left\{f\left(t, y_{k}, y_{k}^{\prime}\right)+B y_{k}^{\prime}(t)+N\left[y_{k+1}(t)-y_{k}(t)\right]-f\left(t, z_{k}, y_{k}^{\prime}\right)\right. \\
& \left.+f\left(t, z_{k}, y_{k}^{\prime}\right)-f\left(t, z_{k}, z_{k}^{\prime}\right)-B z_{k}^{\prime}(t)-N\left[z_{k+1}(t)-z_{k}(t)\right]\right\} \\
\leq & (A+B)^{-1}\left\{-N\left[z_{k}(t)-y_{k}(t)\right]+B\left[z_{k}^{\prime}(t)-y_{k}^{\prime}(t)\right]\right. \\
& \left.+N\left[y_{k+1}(t)-y_{k}(t)-z_{k+1}(t)+z_{k}(t)\right]+B\left[y_{k}^{\prime}(t)-z_{k}^{\prime}(t)\right]\right\} \\
= & (A+B)^{-1} N p(t), \quad t \in J .
\end{aligned}
$$

Thus $y_{k+1}(t) \leq z_{k+1}(t)$ and $y_{k+1}^{\prime}(t) \leq z_{k+1}^{\prime}(t), t \in J$, so (2.2) holds.

Hence, by induction, we have

$$
\begin{cases}y_{0}(t) \leq y_{1}(t) \leq \cdots \leq y_{n}(t) \leq z_{n}(t) \leq \cdots \leq z_{1}(t) \leq z_{0}(t), & t \in J \\ y_{0}^{\prime}(t) \leq y_{1}^{\prime}(t) \leq \cdots \leq y_{n}^{\prime}(t) \leq z_{n}^{\prime}(t) \leq \cdots \leq z_{1}^{\prime}(t) \leq z_{0}^{\prime}(t), & t \in J\end{cases}
$$

for all $n$.

We now show that the sequences $\left\{y_{n}\right\}$ and $\left\{z_{n}\right\}$ converge uniformly and monotonically to $y$ and $z$, respectively, where $y$ and $z$ are solutions of (1.2). The sequences $\left\{y_{n}\right\}$ and $\left\{y_{n}^{\prime}\right\}$ are uniformly bounded because

$$
y_{0}(t) \leq y_{n}(t) \leq z_{0}(t) \quad \text { and } \quad y_{0}^{\prime}(t) \leq y_{n}^{\prime}(t) \leq z_{0}^{\prime}(t), \quad t \in J
$$

for all $n$, where $y_{0}$ and $z_{0} \in C^{1}\left(J, \mathbb{B}^{m}\right)$. Note that the sequences $\left\{y_{n}\right\}$ and $\left\{z_{n}\right\}$ are well-defined because $y_{n}$ and $z_{n}$ are unique solutions of the corresponding linear IVP's. Moreover, $y_{n} \in C^{1}\left(J, \mathbb{R}^{m}\right)$ and

$$
y_{n}(t)=e^{K t}\left[k_{0}+\int_{0}^{t} G_{n-1}(s) d s\right], \quad t \in J,
$$

with $K=(A+B)^{-1} N$ and

$$
G_{j}(s)=e^{-K s}(A+B)^{-1}\left\{f\left(s, y_{j}(s), y_{j}^{\prime}(s)\right)+B y_{j}^{\prime}(s)-N y_{j}(s)\right\} .
$$

It is easy to see that $\left\{y_{n}\right\}$ is a sequence of equicontinuous functions. Indeed, $\left\{z_{n}\right\}$ is a sequence of equicontinuous functions too. 
Note that $\left\{y_{n}^{\prime}\right\}$ and $\left\{z_{n}^{\prime}\right\}$ are sequences of continuous functions on the interval $[0, b]$, so uniform continuity implies that for any $\epsilon>0$ there exists $\delta>0$ such that for all $n$ and $t_{1}, t_{2} \in J$ and $\left|t_{1}-t_{2}\right|<\delta$ we have

$$
\begin{array}{r}
\left\|(A+B)^{-1}\left[f\left(t_{1}, y_{n-1}\left(t_{1}\right), y_{n-1}^{\prime}\left(t_{1}\right)\right)-f\left(t_{2}, y_{n-1}\left(t_{2}\right), y_{n-1}^{\prime}\left(t_{2}\right)\right)\right]\right\|_{*}<\epsilon / 3, \\
\left\|(A+B)^{-1} B\left[y_{n-1}^{\prime}\left(t_{1}\right)-y_{n-1}^{\prime}\left(t_{2}\right)\right]\right\|_{*}<\epsilon / 3, \\
\left\|(A+B)^{-1} N\left[y_{n}\left(t_{1}\right)-y_{n}\left(t_{2}\right)-y_{n-1}\left(t_{1}\right)+y_{n-1}\left(t_{2}\right)\right]\right\|_{*}<\epsilon / 3
\end{array}
$$

because $f$ is continuous on a closed set. Here we used the norm:

$$
\|u\|_{*}=\max _{i=1,2, \ldots, m}\left|u_{i}\right|
$$

From the above and the relation

$$
\begin{aligned}
y_{n}^{\prime}\left(t_{1}\right)-y_{n}^{\prime}\left(t_{2}\right)= & (A+B)^{-1}\left\{f\left(t_{1}, y_{n-1}\left(t_{1}\right), y_{n-1}^{\prime}\left(t_{1}\right)\right)\right. \\
& -f\left(t_{2}, y_{n-1}\left(t_{2}\right), y_{n-1}^{\prime}\left(t_{2}\right)\right)+B\left[y_{n-1}^{\prime}\left(t_{1}\right)-y_{n-1}^{\prime}\left(t_{2}\right)\right] \\
& \left.+N\left[y_{n}\left(t_{1}\right)-y_{n-1}\left(t_{1}\right)-y_{n}\left(t_{2}\right)+y_{n-1}\left(t_{2}\right)\right]\right\}
\end{aligned}
$$

we see that $\left\{y_{n}^{\prime}\right\}$ is a sequence of equicontinuous functions. Hence $y_{n} \rightarrow y, y_{n}^{\prime} \rightarrow y^{\prime}$ and $y \in C^{1}\left(J, \mathbb{R}^{m}\right)$, by Arzeli's theorem. Similarly we have $z_{n} \rightarrow z, z_{n}^{\prime} \rightarrow z^{\prime}$ and $z \in C^{1}\left(J, \mathbb{R}^{m}\right)$. The Lebesgue theorem yields that

$$
\begin{array}{ll}
y(t)=k_{0}+(A+B)^{-1}\left\{\int_{0}^{t}\left[f\left(s, y(s), y^{\prime}(s)\right)+B y^{\prime}(s)\right] d s\right\}, & t \in J, \\
z(t)=k_{0}+(A+B)^{-1}\left\{\int_{0}^{t}\left[f\left(s, z(s), z^{\prime}(s)\right)+B z^{\prime}(s)\right] d s\right\}, & t \in J .
\end{array}
$$

Thus $y$ and $z$ are solutions of problem (1.2).

In the next step we will show that $y$ and $z$ are minimal and maximal solutions of (1.2). Let $x$ be any solution of problem (1.2) such that $y_{0}(t) \leq x(t) \leq z_{0}(t)$ and $y_{0}^{\prime}(t) \leq x^{\prime}(t) \leq z_{0}^{\prime}(t), t \in J$.

We are going to show that

$$
y_{n}(t) \leq x(t) \leq z_{n}(t) \quad \text { and } \quad y_{n}^{\prime}(t) \leq x^{\prime}(t) \leq z_{n}^{\prime}(t), \quad t \in J
$$

for all natural $n$.

Put $p=y_{1}-x$ on $J$. Then

$$
\begin{aligned}
p^{\prime}(t)= & (A+B)^{-1}\left\{f\left(t, y_{0}, y_{0}^{\prime}\right)+B y_{0}^{\prime}(t)+N\left[y_{1}(t)-y_{0}(t)\right]-f\left(t, x, y_{0}^{\prime}\right)\right. \\
& \left.+f\left(t, x, y_{0}^{\prime}\right)-f\left(t, x, x^{\prime}\right)-B x^{\prime}(t)\right\} \\
\leq & (A+B)^{-1}\left\{-N\left[x(t)-y_{0}(t)\right]+B\left[x^{\prime}(t)-y_{0}^{\prime}(t)\right]+B y_{0}^{\prime}(t)\right. \\
& \left.+N\left[y_{1}(t)-y_{0}(t)\right]-B x^{\prime}(t)\right\}=(A+B)^{-1} N p(t), \quad p(0)=0 .
\end{aligned}
$$


Hence $y_{1}(t) \leq x(t)$ and $y_{1}^{\prime}(t) \leq x^{\prime}(t), t \in J$, by Lemma 2.1 .

Let $p=x-z_{1}, t \in J$. Then

$$
\begin{aligned}
p^{\prime}(t)= & (A+B)^{-1}\left\{f\left(t, x, x^{\prime}\right)+B x^{\prime}(t)-f\left(t, z_{0}, x^{\prime}\right)+f\left(t, z_{0}, x^{\prime}\right)\right. \\
& \left.-f\left(t, z_{0}, z_{0}^{\prime}\right)-B z_{0}^{\prime}(t)-N\left[z_{1}(t)-z_{0}(t)\right]\right\} \\
\leq & (A+B)^{-1}\left\{-N\left[z_{0}(t)-x(t)\right]+B\left[z_{0}^{\prime}(t)-x^{\prime}(t)\right]\right. \\
& \left.+B\left[x^{\prime}(t)-z_{0}^{\prime}(t)\right]-N\left[z_{1}(t)-z_{0}(t)\right]\right\} \\
= & (A+B)^{-1} N p(t) .
\end{aligned}
$$

Lemma 2.1 yields $x(t) \leq z_{1}(t)$ and $x^{\prime}(t) \leq z_{1}^{\prime}(t), t \in J$. Thus (2.3) holds for $n=1$. Assume that (2.3) holds for some $k \geq 1$. Put $p=y_{k+1}-x$. Then

$$
\begin{aligned}
p^{\prime}(t)= & (A+B)^{-1}\left\{f\left(t, y_{k}, y_{k}^{\prime}\right)+B y_{k}^{\prime}(t)+N\left[y_{k+1}(t)-y_{k}(t)\right]\right. \\
& \left.-f\left(t, x, y_{k}^{\prime}\right)+f\left(t, x, y_{k}^{\prime}\right)-f\left(t, x, x^{\prime}\right)-B x^{\prime}(t)\right\} \\
\leq & (A+B)^{-1}\left\{-N\left[x(t)-y_{k}(t)\right]+B\left[x^{\prime}(t)-y_{k}^{\prime}(t)\right]+B y_{k}^{\prime}(t)\right. \\
& \left.+N\left[y_{k+1}(t)-y_{k}(t)\right]-B x^{\prime}(t)\right\}=(A+B)^{-1} N p(t), \quad p(0)=0 .
\end{aligned}
$$

Hence $y_{k+1}(t) \leq x(t)$ and $y_{k+1}^{\prime}(t) \leq x^{\prime}(t), t \in J$, by Lemma 2.1 .

Let $p=x-z_{k+1}, t \in J$. Then

$$
\begin{aligned}
p^{\prime}(t)= & (A+B)^{-1}\left\{f\left(t, x, x^{\prime}\right)+B x^{\prime}(t)-f\left(t, z_{k}, x^{\prime}\right)+f\left(t, z_{k}, x^{\prime}\right)\right. \\
& \left.-f\left(t, z_{k}, z_{k}^{\prime}\right)-B z_{k}^{\prime}(t)-N\left[z_{k+1}(t)-z_{k}(t)\right]\right\} \\
\leq & (A+B)^{-1}\left\{-N\left[z_{k}(t)-x(t)\right]+B\left[z_{k}^{\prime}(t)-x^{\prime}(t)\right]\right. \\
& \left.+B\left[x^{\prime}(t)-z_{k}^{\prime}(t)\right]-N\left[z_{k+1}(t)-z_{k}(t)\right]\right\} \\
= & (A+B)^{-1} N p(t) .
\end{aligned}
$$

Lemma 2.1 yields $x(t) \leq z_{k+1}(t)$ and $x^{\prime}(t) \leq z_{k+1}^{\prime}(t), t \in J$. Thus (2.3) holds for all natural $n$.

Now, if $n \rightarrow \infty$, then (2.3) yields $y(t) \leq x(t) \leq z(t)$ and $y^{\prime}(t) \leq x^{\prime}(t) \leq z^{\prime}(t)$, $t \in J$, showing that $y$ and $z$ are minimal and maximal solutions of problem (1.2), respectively.

This ends the proof.

\section{A special case of (1.2)}

Let $m=2$ and $A=\left[\begin{array}{cc}1 & -b \\ 0 & 0\end{array}\right], b \geq 0$. Then problem (1.2) takes the form

$$
\left\{\begin{aligned}
x_{1}^{\prime}(t)-b x_{2}^{\prime}(t) & =f_{1}\left(t, x_{1}(t), x_{2}(t), x_{1}^{\prime}(t), x_{2}^{\prime}(t)\right), & & x_{1}(0)=x_{0,1} \\
0 & =f_{2}\left(t, x_{1}(t), x_{2}(t), x_{1}^{\prime}(t), x_{2}^{\prime}(t)\right), & & x_{2}(0)=x_{0,2}
\end{aligned}\right.
$$


Assume that $f_{1}$ and $f_{2}$ satisfy the following conditions:

$$
f_{i}\left(t, x_{1}, x_{2}, y_{1}, y_{2}\right)-f_{i}\left(t, x_{1}, x_{2}, \bar{y}_{1}, \bar{y}_{2}\right) \leq b_{i, 1}\left[\bar{y}_{1}-y_{1}\right]+b_{i, 2}\left[\bar{y}_{2}-y_{2}\right]
$$

if $\bar{y}_{i} \geq y_{i}$, and

$$
f_{i}\left(t, x_{1}, x_{2}, y_{1}, y_{2}\right)-f_{i}\left(t, \bar{x}_{1}, \bar{x}_{2}, y_{1}, y_{2}\right) \leq-c_{i, 1}\left[\bar{x}_{1}-x_{1}\right]-c_{i, 2}\left[\bar{x}_{2}-x_{2}\right]
$$

if $\bar{x}_{i} \geq x_{i}$ for $i=1,2$ with $b_{i, j}, c_{i, j} \geq 0, i=1,2, j=1,2$.

Note that in this case

$$
\begin{gathered}
B=\left[\begin{array}{ll}
b_{11} & b_{12} \\
b_{21} & b_{22}
\end{array}\right], \quad N=\left[\begin{array}{ll}
c_{11} & c_{12} \\
c_{21} & c_{22}
\end{array}\right], \\
(A+B)^{-1}=\frac{1}{\operatorname{det}(A+B)}\left[\begin{array}{rr}
b_{22} & b-b_{12} \\
-b_{21} & 1+b_{11}
\end{array}\right]
\end{gathered}
$$

provided that $\operatorname{det}(A+B)=b_{22}+b_{21} b+\operatorname{det}(B) \neq 0$. Note that if $b \geq b_{12}$ and $b_{21}=0$, then $(A+B)^{-1} \geq 0$, so assumptions (ii) and (iii) of Theorem 2.2 hold.

The following system

$$
\left\{\begin{aligned}
x_{1}^{\prime}(t) & =f_{1}\left(t, x_{1}(t), x_{2}(t), x_{1}^{\prime}(t)\right), & & x_{1}(0)=x_{0,1} \\
0 & =f_{2}\left(t, x_{1}(t), x_{2}(t), x_{2}^{\prime}(t)\right), & & x_{2}(0)=x_{0,2}
\end{aligned}\right.
$$

is a special case of problem (3.1). Note that in this case we have $b_{12}=b_{21}=0$, so

$$
(A+B)^{-1}=\left[\begin{array}{cc}
1 /\left(1+b_{11}\right) & 0 \\
0 & 1 / b_{22}
\end{array}\right] \text {. }
$$

It is quite simple to formulate corresponding theorems to Theorem 2.2 for problems (3.1) and (3.4).

EXAMPLE. Let us consider the following problem:

$$
\left\{\begin{aligned}
x_{1}^{\prime}(t)-2 x_{2}^{\prime}(t) & =2 x_{1}(t)+3 x_{2}(t)-8\left[x_{1}^{\prime}(t)\right]^{2}-\left[x_{2}^{\prime}(t)\right]^{2}+t, & & t \in J, \\
0 & =x_{1}(t)+\left[x_{1}(t)\right]^{2}+5 x_{2}(t)-10\left[x_{2}^{\prime}(t)\right]^{2}, & & t \in J, \\
x_{1}(0) & =x_{2}(0)=0 . & &
\end{aligned}\right.
$$

Comparing this with (3.1) we have $A=\left[\begin{array}{cc}1 & -2 \\ 0 & 0\end{array}\right], b=2$ and

$$
\begin{aligned}
& f_{1}\left(t, x_{1}, x_{2}, y_{1}, y_{2}\right)=2 x_{1}+3 x_{2}-8 y_{1}^{2}-y_{2}^{2}+t \\
& f_{2}\left(t, x_{1}, x_{2}, y_{1}, y_{2}\right)=x_{1}+x_{1}^{2}+5 x_{2}-10 y_{2}^{2}
\end{aligned}
$$


It is simple to check that

$$
\left\{\begin{array} { l l } 
{ y _ { 0 1 } ( t ) = 0 , } & { t \in J , } \\
{ y _ { 0 2 } ( t ) = 0 , } & { t \in J , }
\end{array} \quad \text { and } \quad \left\{\begin{array}{ll}
z_{01}(t)=t, & t \in J, \\
z_{02}(t)=t, & t \in J
\end{array}\right.\right.
$$

are lower and upper solutions of problem (3.5), respectively.

Let $0 \leq x_{1} \leq \bar{x}_{1} \leq t, 0 \leq x_{2} \leq \bar{x}_{2} \leq t, 0 \leq y_{1} \leq \bar{y}_{1} \leq 1$ and $0 \leq y_{2} \leq \bar{y}_{2} \leq 1$. Then conditions (3.2) and (3.3) hold with

$$
B=\left[\begin{array}{cc}
16 & 2 \\
0 & 20
\end{array}\right] \text { and } N=\left[\begin{array}{ll}
2 & 3 \\
1 & 5
\end{array}\right]
$$

Indeed we have

$$
A+B=\left[\begin{array}{cc}
17 & 0 \\
0 & 20
\end{array}\right] \text { and }(A+B)^{-1}=\frac{1}{340}\left[\begin{array}{cc}
20 & 0 \\
0 & 17
\end{array}\right] \text {. }
$$

Thus all assumptions of Theorem 2.2 are satisfied. By Theorem 2.2, problem (3.5) has minimal and maximal solutions.

\section{References}

[1] R. Bellman, Methods of nonlinear analysis, Volume II (Academic Press, New York, 1973).

[2] T. Jankowski and V. Lakshmikantham, "Monotone iterations for differential equations with a parameter", J. Appl. Math. Stoch. Anal. 10 (1997) 273-278.

[3] G. S. Ladde, V. Lakshmikantham and A. S. Vatsala, Monotone iterative techniques for nonlinear differential equations (Pitman, Boston, 1985).

[4] V. Lakshmikantham and A. S. Vatsala, Generalized quasilinearization for nonlinear problems (Kluwer, Derdrecht, 1998).

[5] J. J. Nieto, "Quadratic approximation of solutions for ordinary differential equations", Bull. Austral. Math. Soc. 55 (1997) 161-168.

[6] J. J. Nieto and N. Alvarez-Noriega, "Periodic boundary value problems for nonlinear first-order ordinary differential equations", Acta Math. Hungar. 71 (1996) 49-58.

[7] Y. Yin, "Remarks on first-order differential equations with anti-periodic boundary conditions", Nonlinear Times and Digest 2 (1995) 83-94.

[8] Y. Yin, "Monotone iterative technique and quasilinearization for some anti-periodic problems", Nonlinear World 3 (1996) 253-266. 